\title{
Temperature dependence of luminescence pecularities in oxygen doped $\mathrm{ZnTe}$ films
}

\author{
N.V. Malushin, V.M. Skobeeva, V.A. Smyntyna \\ Odessa National I.I. Mechnikov University, Research Institute of Physics, 2 Dvoryanskaya str. ONU, 65026 Odessa, Ukraine \\ Phone: +38(482) 230329
}

\begin{abstract}
Temperature dependence of luminescence intensity inherent to zinc telluride films prepared by the method of vacuum deposition and containing an oxygen impurity was investigated. The model explaining non-monotonous behaviour of curve temperature dependence for the "oxygen" band $\left(\lambda_{\max }=650 \mathrm{~nm}\right)$ is offered. According to this model, during quenching luminescence, the centers of a luminescence and those of the majority carriers capture participate. Determined are the values of the activation energy and concentration of the appropriate centers at which abnormal dependence of luminescence intensity on the temperature is observed.
\end{abstract}

Keywords: luminescence, quenching, centre of luminescence, centre of capture, temperature dependence.

Paper received 11.02.03; accepted for publication 16.06.03.

\section{Introduction}

Zink telluride is promising material for creation of light sources in the visible spectrum. Optimisation of its radiative characteristics requires data about parameters of emission centers. One of the methods of determination of energy centers position is based on the measurement of dependence of irradiation on the temperature. The existing models are well applicable to describing the classical temperature quenching of luminescence when with increasing temperature the light intensity exponentially decreases [1]. This paper introduces the model that describes non-monotonous behavior of the temperature dependence of luminescence intensity detected in oxygen doped $\mathrm{ZnTe}$ films $[2,3]$.

\section{Experimental results and discussion}

The luminescence of oxygen doped ZnTe with $\lambda_{\max }=$ $=650 \mathrm{~nm}$ is observed around $77 \mathrm{~K}$. Fig. 1 shows temperature dependence of the luminescence "oxygen" band in $\mathrm{ZnTe}$ films grown by vacuum deposition method in quasi-closed volume under different technological conditions. One can see that with increasing temperature,

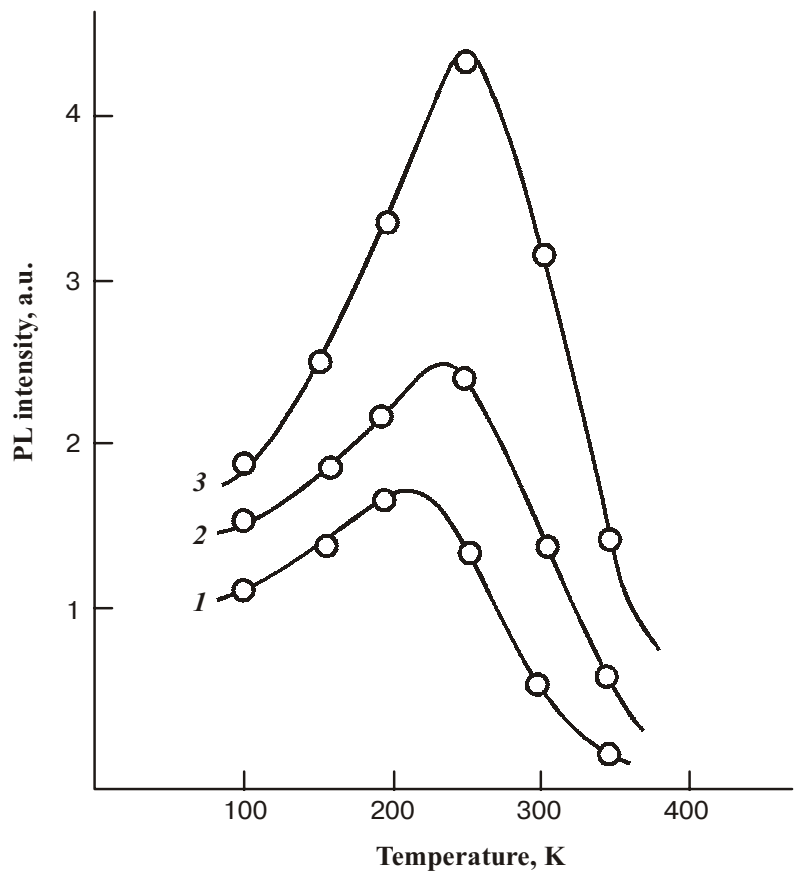

Fig. 1. Temperature dependence of photoluminescence with $\lambda_{\max }=650 \mathrm{~nm}$ of $\mathrm{ZnTe}$ films obtained under different condensation temperatures $\left(T_{k}\right), \mathrm{K}: 570(1) ; 600(2) ; 670(3)$. 


\section{N.V. Malushin et al.: Temperature dependence of luminescence ...}

the flaming up of the luminescence is observed instead of typical quenching. After reaching the maximum, the value of the luminescence intensity falls down, and the value of temperature corresponding to the peak intensity depends on the temperature of film condensation.

It is known [2] that oxygen creates $\mathrm{O}_{\mathrm{Te}}$ defect while replacing the Te atom in $\mathrm{ZnTe}$ lattice, which is the deep donor of the luminescence center. It is remarkable that in the luminescence spectrum of $\mathrm{ZnTe:O}$ only one elementary band is observed, and as implication there are luminescence centers of only one type. It is obvious that for explanation of luminescence quenching mechanism it is necessary to consider the influence of other centers. The role of other centers that take part in the quenching can be played by acceptors located near the valence band. The centers of this kind were observed in $\mathrm{ZnTe}$ under different methods of its preparation. To provide $p$-type of semiconductor conductivity, these centres have different nature and play the role of capture centers for major carriers. The depth of levels of such centers varies from 0.05 to $0.25 \mathrm{eV}$.

In order to explain the temperature dependence of intensive "oxygen" luminescence of the considered $\mathrm{ZnTe}$ samples, the following model was selected.

The presence of two groups of levels in the gap is supposed: acceptor and donor ones with concentrations of respective centres $N_{1}$ and $N_{2}$, and depths $E_{1}$ and $E_{2}$. Under stationary excitation, such system will be described with the following equations

$$
\begin{aligned}
& \gamma_{n_{1}} n p_{1}+\gamma_{p_{1}} p_{1} F e^{-\frac{E_{1}}{k T}}=\gamma_{p_{1}} p\left(N_{1}-p_{1}\right), \\
& \gamma_{p_{2}} p n_{2}+\gamma_{n_{2}} n_{2} F e^{-\frac{E_{2}}{k T}}=\gamma_{n_{2}} n\left(N_{2}-n_{2}\right), \\
& Z=\gamma_{n_{1}} n p_{1}+\gamma_{p_{2}} p n_{2}, \\
& \left(N_{2}-n_{2}\right)-n=\left(N_{1}-p_{1}\right)-p,
\end{aligned}
$$

where $\gamma_{n_{1}}, \gamma_{p_{2}}$ - recombination coefficients of acceptor and donor; $\gamma_{p_{1}}, \gamma_{n_{2}}$ - coefficients of thermal dumping for holes from acceptor and electrons from donor; $p_{1}, n_{2}-$ concentrations of carriers localized on corresponding levels; $p, n$-concentrations of free electrons and holes; $Z$ intensity of stationary photoexcitation; $F$ - effective density of states in the bands.

The model supposes the equality of coefficients of thermal dumping and capturing the carriers on corresponding centers as well as equality of state effective densities for holes and electrons.

In the system of equations (1-4), the first two equations explain the dynamic equilibrium of the carriers exchange process between impurity centers and zones. The equality (3) represents the condition of equilibrium between processes of generation $(Z)$ and recombination of carriers during stationary excitation. The latter equation in the system is the electroneutrality condition.
The system was solved with the following values:

$\gamma_{n_{1}}=10^{-8} \mathrm{~cm}^{3} \mathrm{c}^{-1} \quad E_{1}=0.05 \div 0.25 \mathrm{eV} \quad F=2.5 \cdot 10^{19} \mathrm{~cm}^{-3}$

$\gamma_{p_{2}}=10^{-13} \mathrm{~cm}^{3} \mathrm{c}^{-1} E_{2}=0.1 \div 0.5 \mathrm{eV} \quad Z=10^{15} \mathrm{~cm}^{-3} \mathrm{c}^{-1}$

$\gamma_{n_{2}}=10^{-7} \mathrm{~cm}^{3} \mathrm{c}^{-1} \quad N_{1}=10^{15} \div 10^{16} \mathrm{~cm}^{-3}$

$\gamma_{p_{1}}=10^{-6} \mathrm{~cm}^{3} \mathrm{c}^{-1} \quad N_{2}=10^{14} \div 10^{16} \mathrm{~cm}^{-3}$

The calculated temperature dependence of luminescence intensity can be expressed with the formula

$I_{L}(T)=\gamma_{p 2} p_{(T)} n_{2(T)}$

As expected, at the specific values of $E_{1}, E_{2}, N_{1}$ and $N_{2}$, the calculated dependence $I_{1}(T)$ is non-monotonous. The calculation show that the depth of acceptors bedding $-E_{1}$ and concentration of donors $\left(N_{2}\right)$ have the most considerable influence the temperature curve.

Calculated family of temperature dependence curves at the different values of $E_{1}$ is shown in Fig. 2. One can see that decrease of the value of $E_{1}$ causes the low-temperature-side shift of the maximum of temperature curve. Indeed, the concentration of free carriers increases due to thermal release of acceptor centers, and the closer is this center to the $\mathrm{V}$-band the smaller are the temperatures of the thermal exchange start in this zone.

As a result of donor centers being deep, their energy position does not influence the kind of dependence $I_{1}(T)$. Increasing donor center concentration leads to the following results (see Fig. 3). At the small concentration of $N_{2}$, the dependence $I_{1}(T)$ has the classical shape (curve 1 ), and the energy of activation of the quenching process

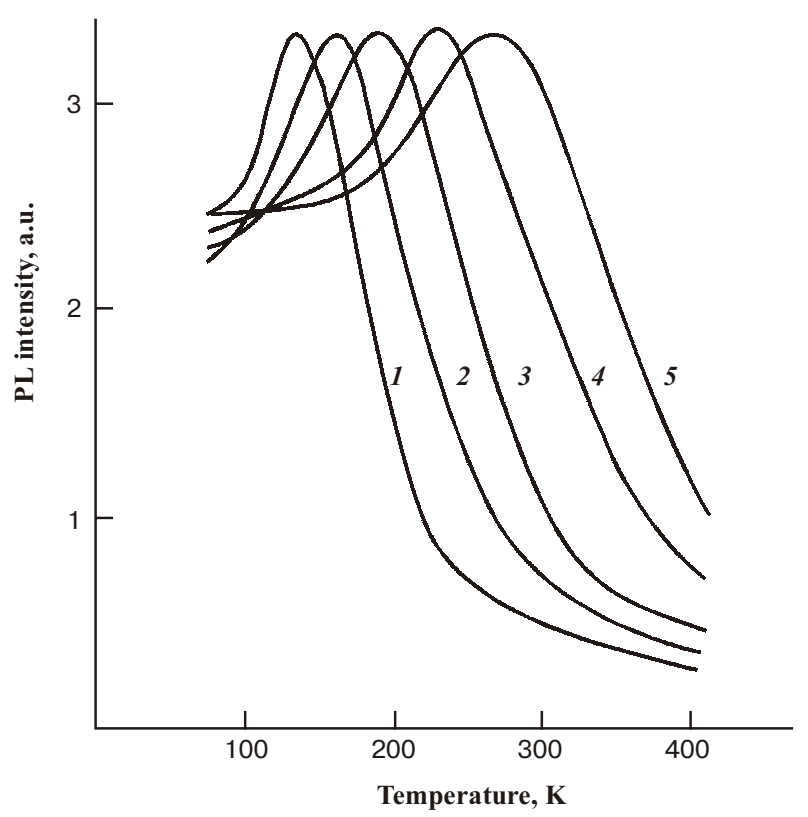

Fig. 2. Theoretical curves of temperature dependences of the luminescence intensity at the different values of $E_{1}, \mathrm{eV}: 1-0.1$; $2-0.12 ; 3-0.14 ; 4-0.17 ; 5-0.2$. 


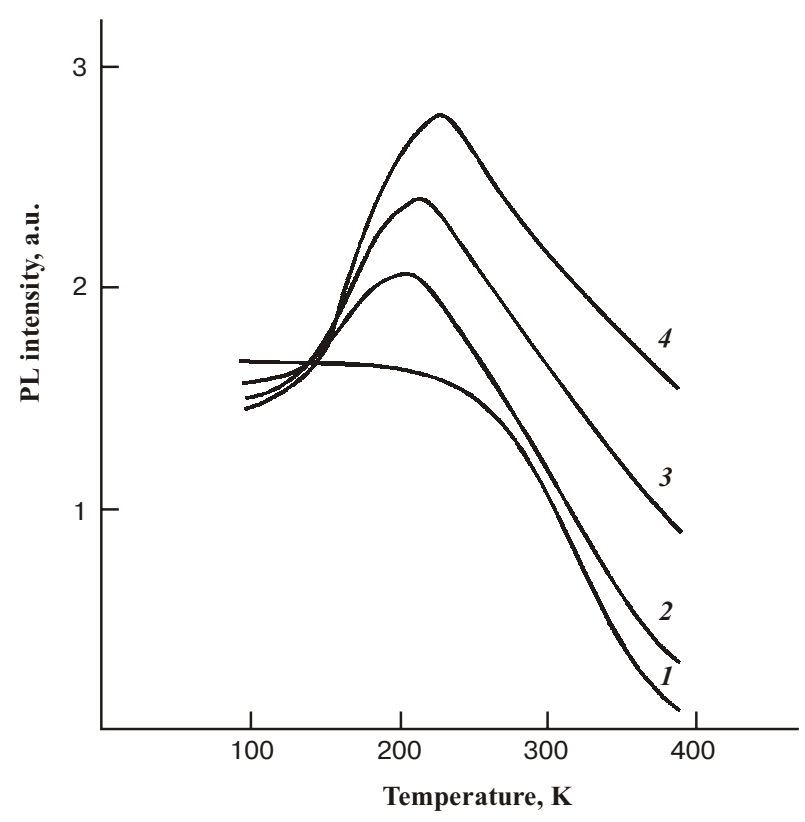

Fig. 3. Theoretical curves for temperature dependences of the luminescence intensity at different concentrations of donor centers $N_{2}: 1-2 \cdot 10^{14} \mathrm{~cm}^{-3}: 2-6 \cdot 10^{14} \mathrm{~cm}^{-3}$.

equals $0.45 \mathrm{eV}$, what correlates with the "oxygen" luminescence center activation energy. Starting from some specific value of $N_{2}$, the temperature dependence of luminescence intensity has a maximum, position of which shifts to the area of high temperatures (curves 2 and 3). This is clear from the following considerations. At the small donor concentration, increase of the free hole concentration caused by their thermal release from acceptor levels influences the temperature dependence only a little, because the intensity of luminescence is controlled by the value $N_{2}$. This influence starts to be noticable at higher values of donor centers. It is also clear that with increase of their concentration luminescence quenching stimulated by thermal release of electrons from donor centers starts at the ever increasing temperatures, what determines the shift of the peak position on the curve $I_{1}(T)$.

In a general case, the peculiarities of temperature dependences $I_{1}(T)$ obtained in the calculations are determined by temperature dependencies of values included in the expressions (5). As computations show, in the temperature interval $100-400 \mathrm{~K}$, the concentration of free holes increases with the temperature, reaching the saturation. In the same temperature interval, with the increase of the temperature, the concentration of electrons on the donor centers in the beginning does not depend on the temperature and then decreases as a consequence of thermal injection in the conductivity band. Superposition of these two processes results in the maximum on the curve $I_{1}(T)$.

\section{Conclusions}

Thus, the proposed model explains the observed temperature dependence of "oxygen" luminescence intensity, and as a result of comparing with theoretically calculated dependence one can determine parameters of centers, which participate in its quenching. For example, for grown ZnTe films the anomaly temperature dependence can be observed at the values: $E_{1}=0.15-0.20 \mathrm{eV} ; E_{2}=$ $=0.45 \mathrm{eV} ; N_{1}=10^{16} \mathrm{~cm}^{-3} ; N_{2}=5 \cdot 10^{14}-15 \cdot 10^{15} \mathrm{~cm}^{-3}$.

\section{References}

1. V.V. Serdyuk, Yu.F. Vaksman, Luminescence of semiconductors (in Russian). Vyshcha chkola, Kiev-Odessa, 1998.

2. N. V. Malushin, V.M. Skobeeva, V.A. Smyntyna, A.K.Dali. Calculation of parameters of "oxygen" centere luminescence in zinc tellurium (in Russian). // The scientific collection Photoelektronika, №.7, p. $44-47$ (1998).

3. A.K.Dali. Determination of ionization optical energy for oxygen in zinc telluride using spectra of luminescence excitation (in Ukrainian). // Ukr. Phys. Journal. 42 (10), p. 12011203 (1997). 